\title{
5
}

\section{Mit welchen Risiken leben wir?}

Jede Technik, sei es zur Energieerzeugung, für die Mobilität, den Warentransport oder für industrielle Prozesse, bringt nicht nur Nutzen, sondern auch Risiken. Wenn der Mensch nicht auf die Vorzüge solcher Technologien verzichten will, muss er lernen, so damit umzugehen, dass er die Kontrolle darüber behält und dass die zusätzlichen Risiken kontrollierbar und so niedrig wie möglich bleiben.

Viele Pannen, Unfälle und Katastrophen sind auf menschliches Fehlverhalten oder Versagen zurückzuführen, seltener auch auf kriminelle Absichten. Der Sicherheitskultur kommt daher oberste Priorität zu. Dazu gehört auch die entsprechende Ausbildung und Motivation der verantwortlichen Mitarbeitenden, verbunden mit einer kontinuierlichen sicherheitstechnischen Überprüfung und Nachrüstung aller technischen Anlagen und Einrichtungen während ihrer gesamten Betriebszeit. Eine wichtige Funktion haben hier auch kompetente und unbestechliche Bewilligungs- und Aufsichtsbehörden.

Andere Ursachen für Störfälle in Verbindung mit technischen Einrichtungen liegen bei Materialfehlern oder Versagen einzelner Komponenten, sie können aber auch durch Naturkatastrophen wie Unwetter, Erdbeben oder Tsunamis ausgelöst oder verstärkt werden, oder durch die Auswirkungen des Klimawandels, der menschengemacht ist.

Die größten Risiken kommen jedoch nach wie vor von der Natur selbst, etwa Naturkatastrophen, Klimaveränderung, Krankheiten, Epidemien und Hungersnöte. Risiken, seien sie naturgegeben oder durch Menschen ver- 
ursacht, sie rechtfertigen denn auch den großen Aufwand in Forschung und Technik, in der Medizin und Pharmaforschung, bei der Katastrophenabwehr und Notfallvorsorge, aber auch bei den Maßnahmen zur Bewältigung der Folgen solcher Ereignisse und bei der Stärkung der Resilienz bei den betroffenen Kollektiven.

\subsection{Risiken der Technik und Risikowahrnehmung}

\subsubsection{Wir leben beständig mit zivilisationsbedingten Risiken}

Ist die Kernenergie nun gefährlicher als andere Methoden zur Energiegewinnung oder andere technische Prozesse? Ein solcher Vergleich ist nicht einfach, da die Wahrnehmung tödlicher Auswirkungen eines Kernkraftwerkunfalls eine andere ist als die langfristigen, ebenfalls tödlichen Folgen der klassischen Energiegewinnung aus fossilen Brenn- und Treibstoffen, wie etwa der Stromproduktion mit Kohle. An einigen Beispielen soll gezeigt werden, dass letztere wesentliche gravierendere Auswirkungen auf Mensch und Umwelt hat.

Unfälle bei großtechnischen Anlagen können verheerende Wirkungen auf die belebte und unbelebte Umwelt haben: Zahlreiche Tankerunfälle ${ }^{1}$ oder die Katastrophe mit der Ölplattform „Deepwater Horizon“ im Golf von Mexiko im Jahr 2010; Staudammbrüche ${ }^{2}$, die allein im 20. Jahrhundert weltweit eine Viertel Million Tote forderten [23]; die Chemiekatastrophe von 1984 bei Bhopal in Indien mit zwischen 3800 und 25.000 Toten sowie einer halben Million Verletzten [22]; auch der Bergbau forderte im 19. Jahrhundert gegen 5000 Tote, im 20. Jahrhundert gar $15.000[25]$.

\footnotetext{
${ }^{1}$ Beispielsweise die Santa-Barbara-Ölpest von 1969, bei der 14.000 t Rohöl ausliefen und die Küste von Südkalifornien verschmutzten, oder das Exxon-Valdes-Tankerunglück, bei dem vor der Küste Alaskas 37.000 t Rohöl ausflossen.

${ }^{2}$ Beispiele sind der Bruch des Staudammes von Vajont, Italien, im Jahre 1963, der 2000 Todesopfer forderte, oder das Versagen der Banqiao-Staudammanlage in China vom August 1975 mit 26.000 bis eventuell gar 85.000 unmittelbaren Todesopfern sowie ca. 145.000 Toten (je nach Quelle 86.000 bis 230.000) durch anschließende Epidemien und Hungersnöte.
} 


\subsubsection{Die Risiken der Stromerzeugung}

Die Auswirkungen verschiedener Verfahren der Stromerzeugung wurden in mehreren Studien untersucht; einige Ergebnisse seien hier zusammengefasst. Die meisten Untersuchungen berechnen die Anzahl erwarteter Todesfälle pro Energieeinheit, meist pro erzeugte Kilowattstunde $(\mathrm{kWh})$. Nur wenige Studien befassen sich auch mit den Auswirkungen auf die Natur oder auf die Gesundheit der betroffenen Bevölkerung.

Abb. 5.1 zeigt die Ergebnisse zweier Studien zur Anzahl der Todesfälle pro TWh (Milliarden kWh) erzeugter Energie durch verschiedene Verfahren der Stromgewinnung. Die fossilen Energien stehen dabei an der Spitze, denn fossile Brenn- und Treibstoffe haben durch die freigesetzten Schadstoffe die weitaus gravierendsten Auswirkungen auf Mensch, Umwelt und Klima. Die Kernenergie schneidet bei diesen Studien um zwei bis drei Größenordnungen besser ab. Hierbei sind neben dem Normalbetrieb auch die schweren Unfälle Tschernobyl und Fukushima berücksichtigt. Aber auch die Stromproduktion mittels Biomasse, Sonne und Wind ist nicht risikofrei, so u. a. durch Gefahren bei der Herstellung dieser Anlagen, bei der Beschaffung der

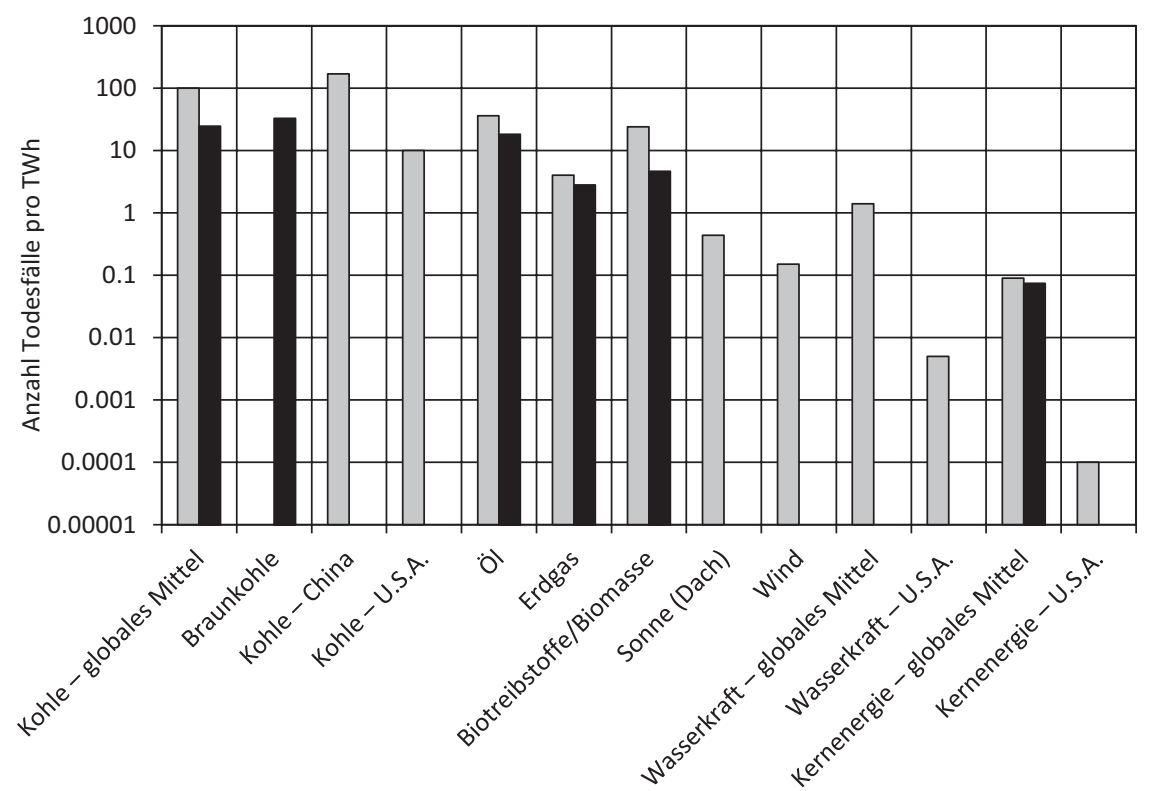

Abb. 5.1 Berechnete Anzahl der Todesfälle pro TWh $\left(10^{9} \mathrm{kWh}\right)$ erzeugter elektrischer Energie durch verschiedene Verfahren der Stromproduktion; grau: nach [5], schwarz: nach [29] 
hierzu benötigten Rohstoffe und Materialen, bei Transport und Betrieb, bei Pannen, Unterhalt und Rückbau. Letztlich gilt das jedoch für alle Verfahren zur Energiegewinnung.

Die Ergebnisse solcher Studien hängen wesentlich von den verwendeten Rohdaten und Randbedingungen ab, sowie der Art der Anlagen und dem Land, wo diese betrieben werden. Deshalb sollten die Resultate eher verwendet werden, um die Größenordnung aufzuzeigen und einen relativen Vergleich zu ermöglichen.

\subsubsection{Die Freisetzung von $\mathrm{CO}_{2}$ und anderen Treibhausgasen}

Maßgeblich für die Auswirkungen auf den Klimawandel sind die Emissionen von Treibhausgasen. $\mathrm{Zu}$ diesen zählen Wasserdampf, $\mathrm{CO}_{2}, \mathrm{CH}_{4}, \mathrm{NO}_{\mathrm{x}}$, FCKW, $\mathrm{SF}_{6}, \mathrm{O}_{3}$ und $\mathrm{NF}_{3}$. Eine umfassende Bilanz der schweizerischen Treibhausgasemissionen wurde von einer Arbeitsgruppe im Auftrag des Schweizer Departementes für Umwelt, Verkehr, Energie und Kommunikation zusammengestellt [33]. Hieraus werden im Folgenden einige Ergebnisse zitiert und in Abb. 5.2 dargestellt. Um alle wichtigen Treibhausgase entsprechend ihrer Auswirkungen auf das Klima zu berücksichtigen, werden diese in $\mathrm{CO}_{2}$-Äquivalent angegeben.

Der $\mathrm{CO}_{2}$-Ausstoß der Schweiz betrug 2015 38,85 Megatonnen (Mt) $\mathrm{CO}_{2}$ pro Jahr. Werden noch die wichtigsten der übrigen Treibhausgase $\left(\mathrm{CH}_{4}, \mathrm{NO}_{\mathrm{x}}\right.$ und synthetische Gase) hinzugezählt, ergibt sich ein Total von 48,14 $\mathrm{Mt} \mathrm{CO}_{2}$-Äquivalent. Das ist allerdings nur die halbe Wahrheit, denn es wird auch $\mathrm{CO}_{2}$ in Form von Produkten oder Dienstleistungen exportiert oder importiert ("graue»Treibhausgase). Der Import überwiegt um 46,2 Mt $\mathrm{CO}_{2}$-Äquivalent (2011) und ergibt so einen Gesamtwert von $95 \mathrm{Mt}$ $\mathrm{CO}_{2}$-Äquivalent, also 11,3 t pro Kopf. Seit 1990 wird eine leichte Abnahme um 0,4 Mt $\mathrm{CO}_{2}$-Äquivalent pro Jahr beobachtet, auch der Pro Kopf-Ausstoß an Treibhausgasen sank in der Schweiz von 1990 bis 2015 um etwa einen Viertel, wohingegen das BIP seit 1990 um 45 \% und die Bevölkerung um $24 \%$ (im Schnitt 1,5\% pro Jahr) anstieg. Der Anteil des Verkehrs nahm nur leicht zu, obwohl die Anzahl der Fahrzeuge (PW) seit 2000 um jährlich 1,7\% zunimmt. Infolge der besseren Energieeffizienz moderner Autos sinkt der $\mathrm{CO}_{2}$-Ausstoß pro Fahrzeugleistung. Derselbe Trend wird auch beim Güterverkehr auf der Straße beobachtet. Erwähnenswert ist auch, dass beim $\mathrm{CO}_{2}$-Ausstoß des Verkehrs der Dieselanteil zwischen 1990 und 2015 von rund 20 auf über $50 \%$ angestiegen ist. Beim Flugverkehr ist der 
a

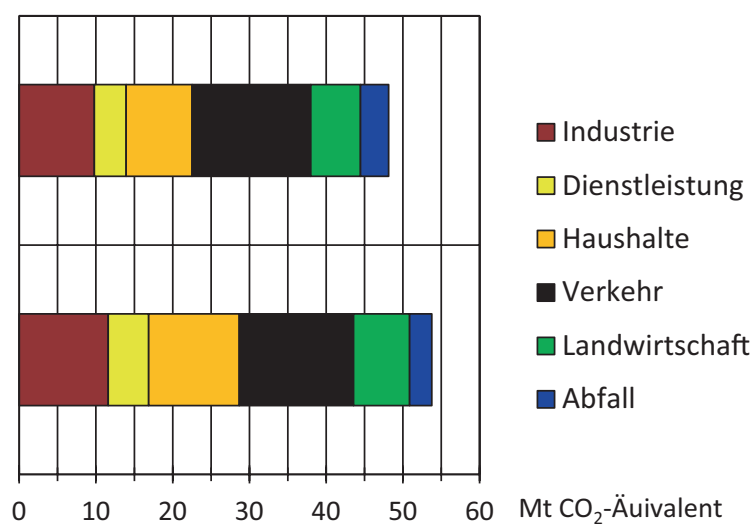

b

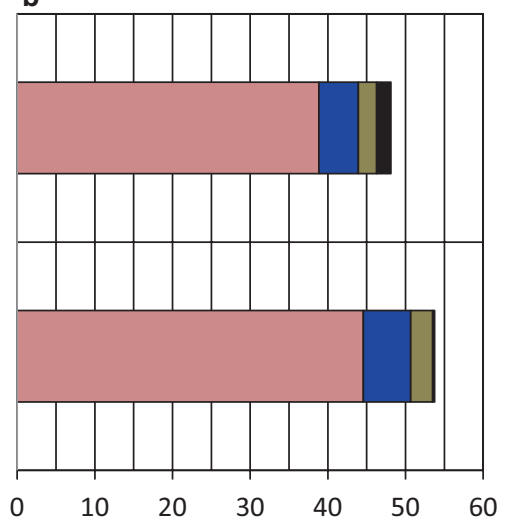

$\square \mathrm{CO} 2$

$\square$ Methan

$\square$ Stickoxide

synthetische Treibhausgase

Abb. 5.2 Treibhausgasemissionen der Schweiz in $\mathrm{Mt} \mathrm{CO}_{2}$-Äquivalent nach Quellen (a) und nach Treibhausgasen (b): für die Jahre 1990 (b) und 2015 (a) [33]

$\mathrm{CO}_{2}$-Ausstoß nur gering angestiegen, obwohl die Verkehrsleistung von 1990 auf 2014 auf $220 \%$ zugenommen hat [33]. Eine Besteuerung des Kerosins sowie eine umweltgerechte $\mathrm{CO}_{2}$-Abgabe der Zivilluftfahrt ist jetzt in Diskussion und wird hoffentlich auch bald eingeführt.

Im Ländervergleich betragen die $\mathrm{CO}_{2}$-Emissionen (ebenfalls in $\mathrm{Mt}^{\mathrm{CO}_{2}}$ pro Jahr, sowie in Klammern in Tonnen $\mathrm{CO}_{2}$ pro Einwohner und Jahr): Australien: 400 (16,6); China: 10.357 (7,5); Deutschland: 798 (9,9); Frankreich: 340 (5,3); Indien: 2.274 (1,73); Italien: $361(6,0)$; Japan: 237 (9,8); Kanada: 557 (15,5); Österreich: 67 (7,9); Polen: 316 (8,2); Russland: 617 (11,3); Schweiz: $40(4,8)$ und USA: $414(16,9)$ [11]. Im Vergleich zu den übrigen Industrienationen schneidet hier die Schweiz, da sie fast keinen Strom aus fossilen Energieträgern bezieht, relativ gut ab. Die rasch 


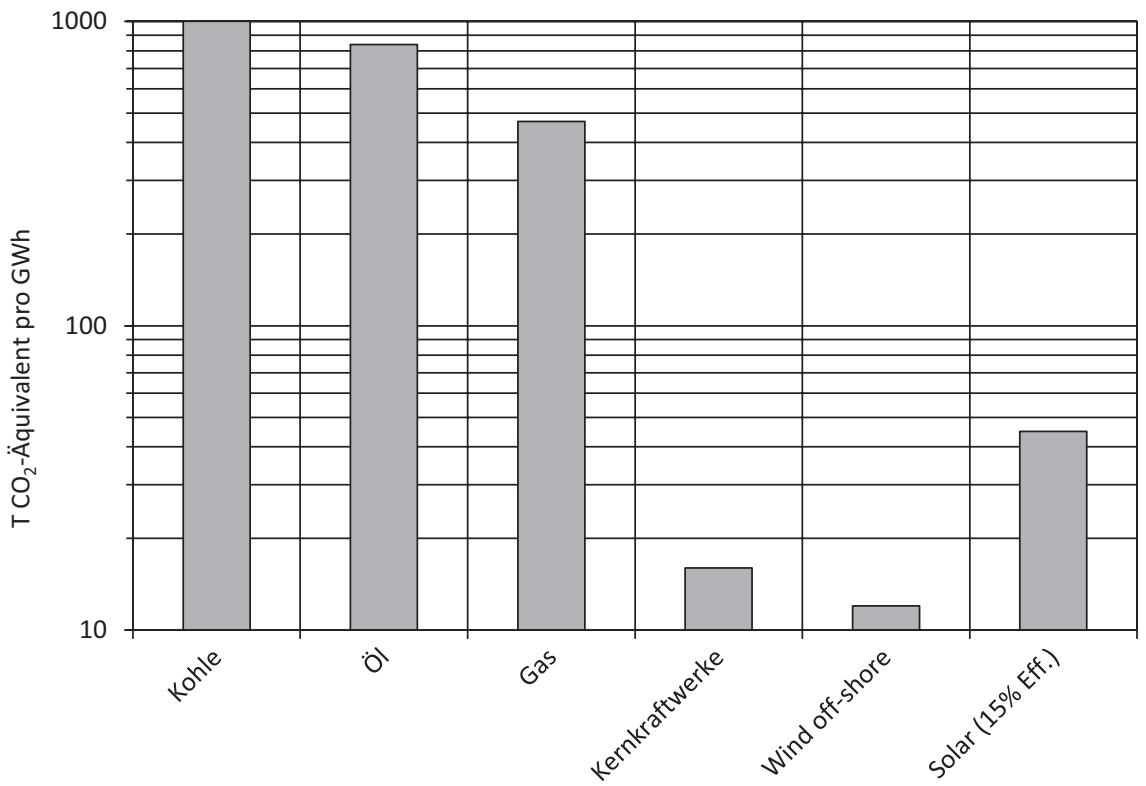

Abb. $5.3 \mathrm{CO}_{2}$-Emissionen von verschiedenen Verfahren zur Stromerzeugung in $\mathrm{g}$ $\mathrm{CO}_{2}$-Äquivalent pro kWh. (Nach [36], S. 155)

wachsenden Volkswirtschaften in China und Indien haben bei diesen beiden Staaten zu einem deutlichen Anstieg des $\mathrm{CO}_{2}$-Ausstoßes um über $400 \%$ zwischen 1990 und 2017 geführt [33].

Abb. 5.3 zeigt die $\mathrm{CO}_{2}$-Freisetzung bei verschiedenen Verfahren der Stromerzeugung, angegeben in $\mathrm{g} \mathrm{CO}_{2}$-Äquivalent pro $\mathrm{kWh}$. Im Hinblick auf den Klimawandel ist hier der Vorteil der Kernenergie gegenüber den fossilen Energien deutlich zu erkennen. Würden die Schweizer den Kernenergieanteil von $36 \%$ an ihrer derzeitigen Stromproduktion (2016) durch $\mathrm{GuD}$, beziehungsweise durch Kohle-Kraftwerke ersetzen, ergäben sich zusätzliche $\mathrm{CO}_{2}$-Emissionen von rund 20 respektive von über $50 \mathrm{Mt} \mathrm{CO}_{2}$ pro Jahr.

\subsubsection{Unsere täglichen Risiken und deren Wahrnehmung}

Bei der Wahrnehmung der Risiken ist der Mensch leider nicht sehr objektiv. Ein Beispiel möge dies erläutern: In einen Großbetrieb mit 100.000 Mitarbeitenden ereigneten sich jedes Jahr einige kleine Unfälle mit jeweils wenigen Verletzten und pro Jahr einem Todesfall. Das ergibt für Mitarbeitenden 


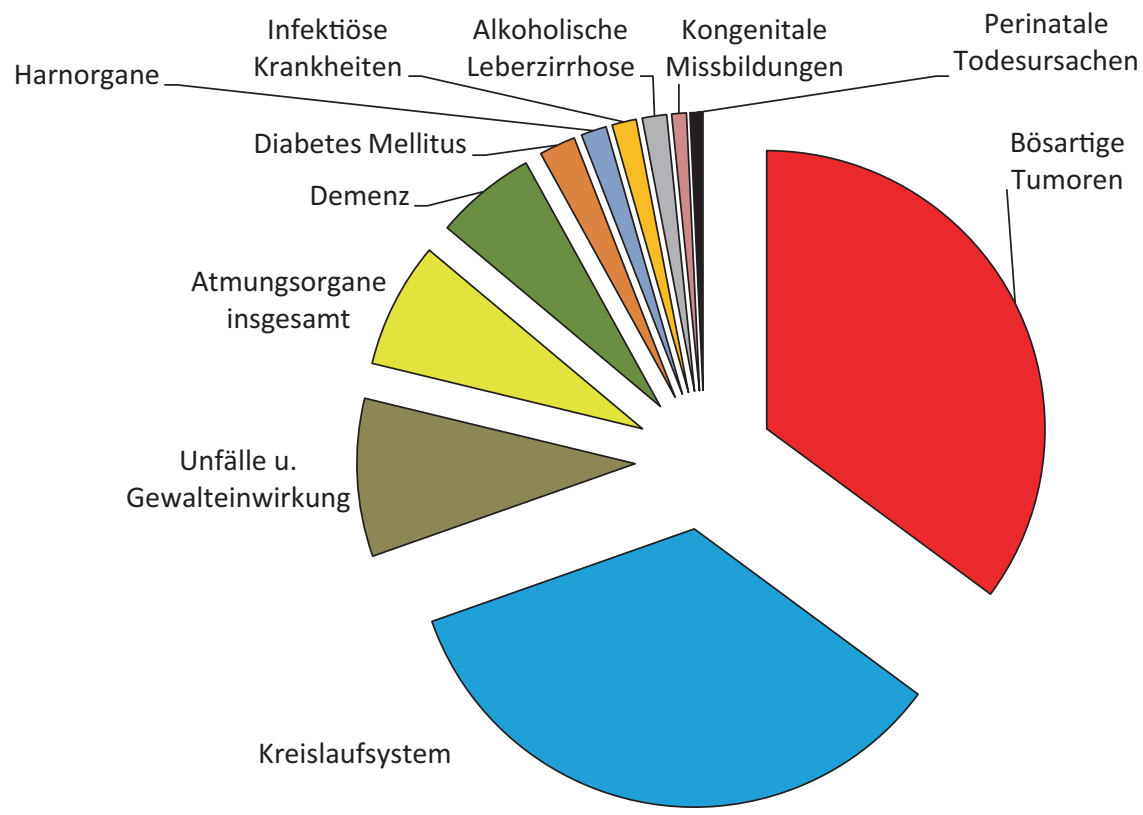

Abb. 5.4 Woran sterben die Schweizer? Altersstandardisiertes Sterberisiko der Schweizer Bevölkerung, gemittelt über die Jahre 2010-2015 (Spezifische Todesursachen Schweiz, [1, 2])

diese Firma ein durchschnittliches Berufsrisiko von $10^{-5}$ pro Jahr. Ein hypothetisches Großereignis in demselben Betrieb, das nur alle 100 Jahre einmal vorkommt und 100 Todesfälle zur Folge hätte (und somit ebenfalls zu einem Risiko von $10^{-5}$ pro Jahr führt), wird subjektiv als gravierender empfunden als die kleinen Unfälle. Dies liegt wohl daran, dass eine plötzliche Veränderung mit großem Schaden anders wahrgenommen wird als ein Schadensausmaß gleicher Größe, das sich allerdings über einen längeren Zeitraum in kaum wahrnehmbaren kleinen Schritten aufsummiert.

Die Sterberisiken für die Schweizer Bevölkerung (Abb. 5.4 und Abb. 5.5) werden durch verschiedene Faktoren bestimmt: Das Alter, das eigenes Verhalten, dasjenige unserer Mitmenschen, Lebens- und Ernährungsgewohnheiten, Beruf und Freizeitgestaltung, aber auch durch Natur und Umwelt sowie Einflüsse von Genetik und Epigenetik ${ }^{3}$ und, nicht zu vergessen, durch den Zufall. Auf Genetik und Zufall die haben wir keinen Einfluss;

\footnotetext{
${ }^{3}$ Der Begriff Epigenetik umschreibt Mechanismen und Konsequenzen vererbbarer ChromosomenModifikationen, die nicht auf Veränderungen der DNA-Sequenz beruhen, sondern von Umwelt und Lebenswandel beeinfluss werden.
} 

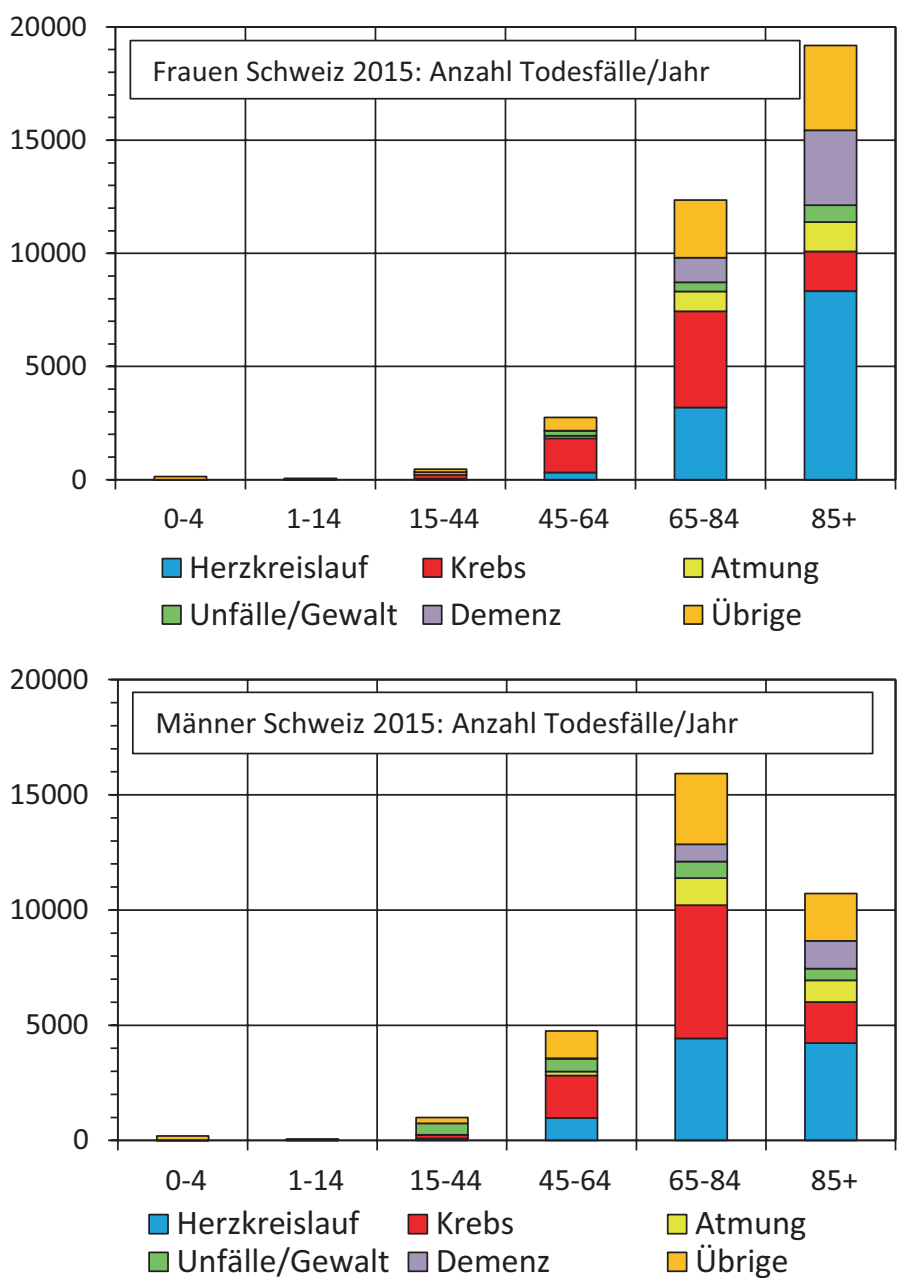

Abb. 5.5 Sterbezahlen der Schweizer Bevölkerung nach Alterskategorien: Anzahl der Sterbefälle pro Jahr, nach Ursache und Altersklasse [1, 2]

bei den anderen Faktoren schon, aber in unterschiedlichem Ausmaß. Während wir bei Umwelt und Epigenetik in erster Linie vom Verhalten unserer Umwelt abhängig sind, spielt bei den übrigen Ursachen unser eigenes Verhalten eine Rolle [30]. Durch dieses setzen wir uns selbst, aber auch unsere Mitmenschen, Risiken aus. Gegen die Bedrohung aus der Natur treffen wir umfangreiche Maßnahmen, etwa bei Gefahrenabwehr und -bewältigung, bei der Stärkung der Resilienz (der Fähigkeit, rasch zur Normalität zurückzukehren) und durch vorbeugenden Maßnahmen wie medizinische Therapien. 


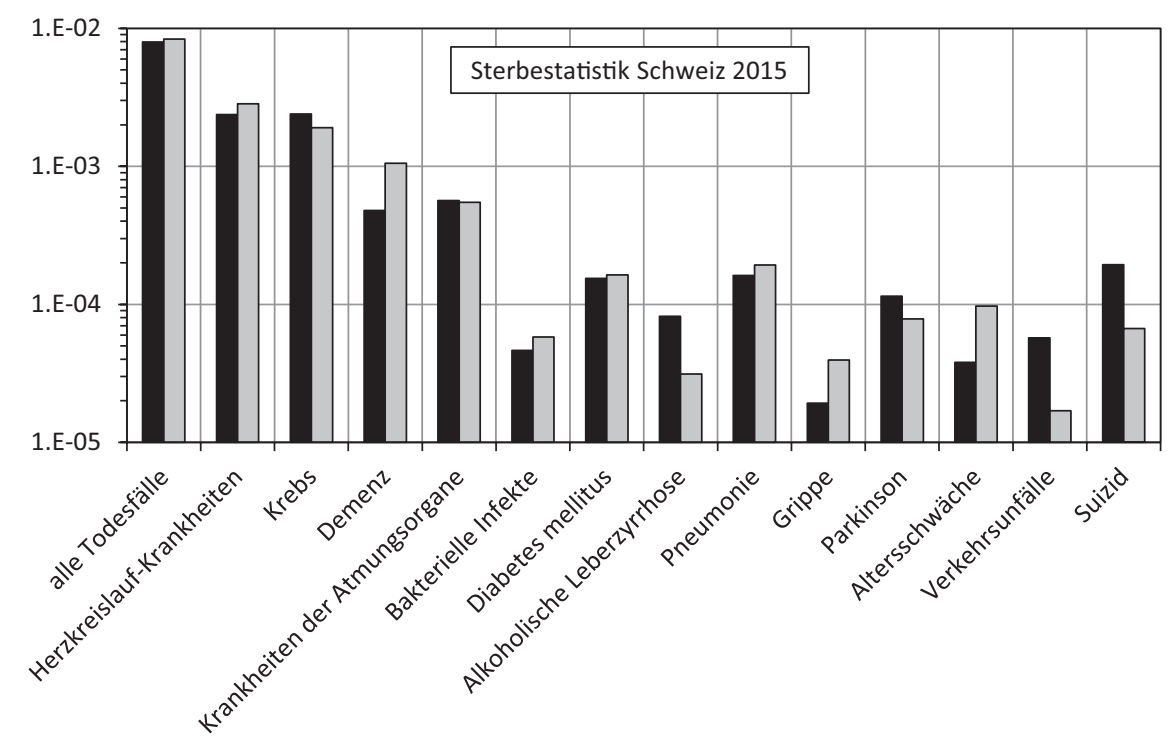

Männer (2015) Sterberisiko/Jahr $\quad \square$ Frauen (2015) Sterberisiko/Jahr

Abb. 5.6 Sterbewahrscheinlichkeit pro Jahr der Schweizer Bevölkerung nach Geschlecht und Ursache (2015). (Nach [1-3])

Als weiteren Vergleich zeigt Abb. 5.6 die Risiken des täglichen Lebens der Schweizer Bevölkerung. Die hier zitierten Zahlenwerte sind Durchschnittswerte; sie hängen ab von Wohnort, Personengruppe und Altersklasse. In der Jugend dominieren Todesfälle durch Verkehrs- und andere Unfälle sowie durch Infektionskrankheiten; im mittleren bis hohen Alter überwiegen jene durch Haushaltsunfälle, Krebs- sowie Herz-Kreislauf-Erkrankungen, in höherem Alter vermehrt auch Demenz [30]. ${ }^{4}$

Jeder Einzelne kann durch sein Verhalten im Haushalt und im Straßenverkehr, in seinem Beruf und bei seiner Freizeitgestaltung sein Risiko beeinflussen. Auch Lebens- und Ernährungsgewohnheiten spielen eine große Rolle (Rauchen, Konsum von Alkohol und Suchtstoffen, Übergewicht). Da mit fortgeschrittenem Alter Krebs- und Herzkreislauferkrankungen zunehmen, können vorbeugende Untersuchungen (bei Frauen Brust, bei Männern Prostata; bei beiden Geschlechtern Darm, Blutdruck, Cholesterin, etc.) und rechtzeitig ergriffene medizinische Vorsorgemaßnahmen das

\footnotetext{
${ }^{4}$ Für Deutschland (2010) beträgt das jährliche Sterberisiko durch Unfälle: Arbeit: $6,4 \cdot 10^{-6}$, Verkehr: 4,6 $\cdot 10^{-5}$, Schule: $1,5 \cdot 10^{-7}$, Hausbereich: $9,1 \cdot 10^{-5}$, Freizeit: $8,8 \cdot 10^{-5}$ und sonstige: $1,3 \cdot 10^{-5}([30]$.
} 
Tab. 5.1 Risikovergleiche durch Strahlung unter Benutzung der ICRP-Risikofaktoren [15]

\begin{tabular}{llll}
\hline Expositionssituation & $\begin{array}{l}\text { Dosis } \\
\text { mSv/Jahr }\end{array}$ & $\begin{array}{l}\text { Krebsrisiko } \\
\text { pro Jahr }^{a}\end{array}$ & $\begin{array}{l}\text { Erhöhung gegenüber } \\
\text { natürlichem Risko }\end{array}$ \\
\hline $\begin{array}{l}\text { natürlichen externe Strahlen- } \\
\text { exposition der Schweizer }\end{array}$ & 1 & $5 \cdot 10^{-5}$ & $1,7 \%$ \\
$\begin{array}{l}\text { Bevölkerung } \\
\begin{array}{l}\text { Zusätzliche Strahlenexposition in der } \\
\text { Nahumgebung des Schweizer Kern- } \\
\text { kraftwerk Gösgen/CH }\end{array}\end{array}$ & $0,000.3$ & $1,5 \cdot 10^{-8}$ & $0,000.5 \%$ \\
$\begin{array}{c}\text { Durchschnittliche beruflich Strahlen- } \\
\text { exposition in Schweizer Kernenergie } \\
\text { Durchschnittliche Exposition durch } \\
\text { Radon im Wohn- und Arbeitsbereich } \\
\text { in der Schweiz }\end{array}$ & 0,06 & $2,4 \cdot 10^{-6}$ & $0,08 \%$ \\
\hline
\end{tabular}

${ }^{a}$ Das spontane Krebssterberisiko der Schweiz beträgt rund $3 \cdot 10^{-3} / \mathrm{Jahr}$ ${ }^{b}$ Hier nur Lungenkrebssterblichkeit

Sterberisiko reduzieren. Im Hinblick auf eine persönliche Risikooptimierung ist es daher nützlich, seine eigenen Risiken und deren Ursachen zu kennen und zur Senkung des Gesamtrisikos zuerst bei den größten Risiken Maßnahmen zu ergreifen.

\section{Subjektive Risikowahrnehmung beim Vergleich von Flug- und Straßenverkehr}

Bei Verkehrsunfällen sterben nach Angaben der WHO weltweit jedes Jahr etwa 1,4 Mio. Menschen (in Europa 26.000). Die hauptsächlich durch den Straßenverkehr verursachte Luftverschmutzung fordert zusätzlich jedes Jahr mehrere Millionen Menschenleben. Der zivile Luftverkehr ${ }^{5}$, der subjektiv von vielen als unsicherer als der Straßenverkehr wahrgenommen wird, verursachte zwischen 1959 und 2013 weltweit insgesamt 30.566 Todesfälle, also im Schnitt «nur» einige Hundert pro Jahr, und dies mit deutlich abnehmender Tendenz [31, 34]. Der Luftverkehr ist also um Größenordnungen sicherer als der Straßenverkehr.

\footnotetext{
${ }^{5} 2015$ starben bei Flugunfällen 452 Menschen. Darin inbegriffen sind zwei große Unfälle, die durch menschliches Einwirken willentlich verursacht wurden: Der Germanwings-Absturz in den Pyrenäen vom 24.03.2015, verursacht durch den Selbstmord des Co-Piloten, forderte 150 Todesopfer; beim Abschuss eines russischen Airbus A321 des Metro-Jet-Charterflugs vom 31.10.2015 durch eine Rakete über der Sinai-Halbinsel kamen 224 Menschen ums Leben. Für die Jahre 2011 bis 2018 werden in der zitierten Quelle die folgenden Zahlen angegeben: 2011: 432, 2012: 318, 2013: 218, 2014: 814, 2015: 542, 2016: 332, 2017: 57 und 2018: 779 Tote durch Flugzeugabstürze weltweit [24, 31].
} 
Das Risiko durch Strahlung, berechnet mit den ICRP-Risikofaktoren, ist in Tab. 5.1 anhand von vier Beispielen dargelegt. Das sich daraus ergebenden zusätzliche jährliche Krebssterberisiko wird mit dem spontanen Sterberisiko durch Krebs verglichen.

Solchen Risikovergleiche belegen, dass Maßnahmen im Bereich von Strahlendosen von deutlich unter einem $1 \mathrm{mSv}$ pro Jahr mit dem Ziel, diese und deren Risiken noch weiter zu senken, keinen wesentlichen Einfluss auf das Gesamtrisiko haben.

\subsection{Die Kohleverstromung in Europa}

Ein im Sommer 2016 erschienener Bericht befasst sich mit den gesundheitlichen Auswirkungen der Kohlekraftwerke in Europa [7, 8]. Er zeigt auf, wie stark Emissionen aus Kohlekraftwerken die menschliche Gesundheit beeinträchtigen. Bei den europäischen Kohlekraftwerken handelt es sich meist um ältere Anlagen, die noch nicht mit Partikelfiltern und Abgasreinigungsanlagen nachgerüstet wurden. Die Studie ergibt, dass die 257 europäischen Kohlekraftwerke durch Feinstaub, Schwefeldioxid $\left(\mathrm{SO}_{2}\right)$, Stickoxide $\left(\mathrm{NO}_{\mathrm{X}}\right)$ und Quecksilber in ihren Abgasen europaweit jährlich 22.900 Todesfälle (95\% Vertrauensbereich von 14.400 bis 31.900) verursachen; das entspricht in etwa den Gefahren durch den Straßenverkehr, der in Europa jährlich 26.000 Todesopfer fordert. Neben den Todesfällen treten Lungenerkrankungen auf wie chronische Bronchitis, Chronic obstructive pulmonary disease (COPD) und Asthma, was zu Arbeitsausfällen führt und das Gesundheitssystem belastet. Der europaweit entstehende volkswirtschaftliche Schaden wird auf 32 bis $62 \mathrm{Mrd}$. € geschätzt.

\footnotetext{
Alle 9 Monate ein Tschernobyl?

Der folgende Vergleich mit Tschernobyl mag etwas gewagt erscheinen, der Leser möge dennoch weiterlesen! Der Reaktorunfall von Tschernobyl war eine schreckliche Katastrophe. Er hätte vermieden werden können, wenn die Anlage regelmäßig sicherheitstechnisch überprüft und nachgerüstet worden wäre.

Die wohl seriöseste Studie über die zu erwartenden Krebstodesfälle durch Tschernobyl ist diejenige der Internationalen Agentur für Krebsforschung IARC in Lyon [4], ${ }^{6}$ die in Zusammenarbeit mit der WHO realisiert wurde. Sie rechnet für ganz Europa und für einen Zeitraum von 80 Jahren mit 16.000 zusätzlichen, strahlenbedingten Krebstodesfällen (bei einem 95-Prozent-Vertrauensbereich von 3400 bis 72.000). Andere Studien kommen zu leicht höheren Zahlen.
}

${ }^{6}$ Zur Erläuterung dieser Zahlenwerte siehe Kap. 2 

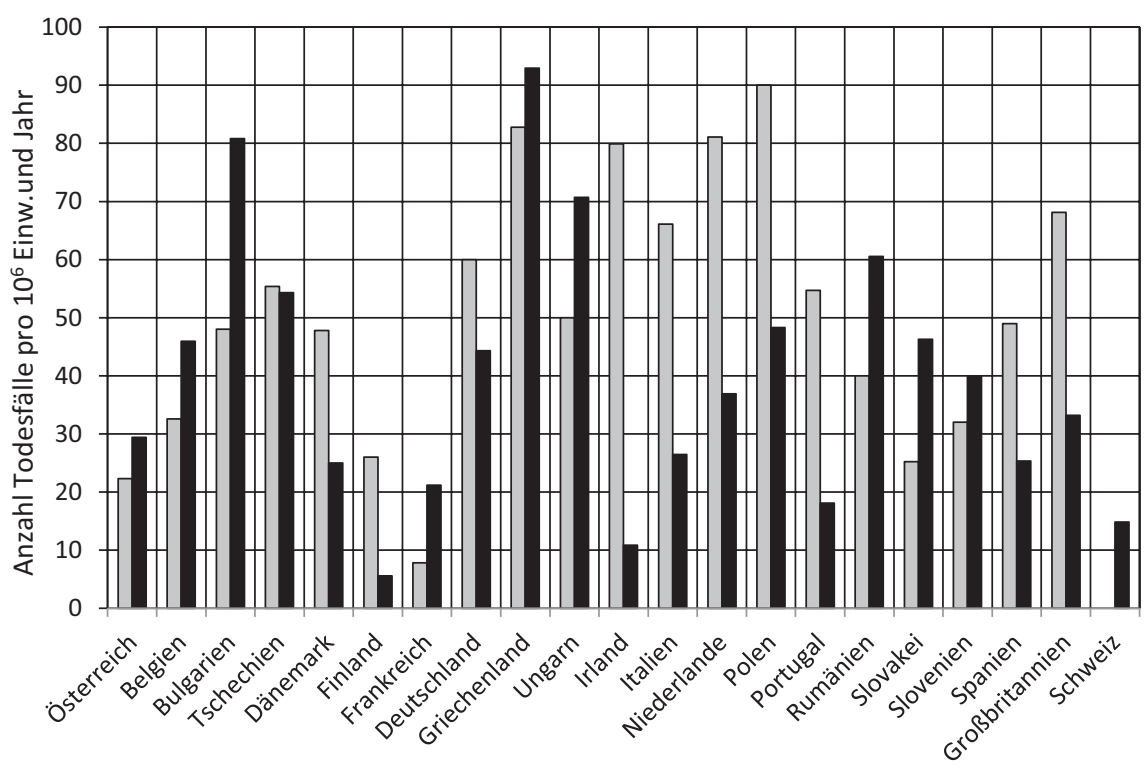

Abb. 5.7 Durch europäische Kohlekraftwerke verursachtes, berechnetes Sterberisiko in Anzahl Fälle pro Million Einwohner und Jahr (schwarz) im Vergleich zum prozentualen Anteil an Strom aus fossilen Energiequellen (grau) nach Ländern. (Nach $[7,8]$ )

Im gleichen Zeitraum werden die europäischen Kohlekraftwerke gemäß der genannten Studie Europe's Dark Cloud (sofern diese weiterhin auf heutigem technischem Stand verbleiben) 1,8 Mio. zusätzliche Krebstodesfälle verursachen, also 110-mal mehr als durch Tschernobyl.

Beschränkt man sich auf die Anzahl Todesfälle durch Krebs, ist die Kohleverstromung in Europa somit gleichbedeutend mit «ein Tschernobyl alle 9 Monate».

Die Ergebnisse der Studie Europe's Dark Cloud sind in Abb. 5.7 nach Ländern aufgeschlüsselt, in schwarz die Anzahl Todesfälle durch Kohleverstromung pro Million Einwohner, in grau der Anteil des $\mathrm{CO}_{2}$-belasteten Stroms des betreffenden Landes. Ein Zusammenhang zwischen dem prozentualen Anteil an fossilem Strom und dem Sterberisko durch Kohleverstromung ist bei Ländern zu erkennen, deren Nachbarländer ebenfalls Kohle in großer Menge verstromen (A, B, BG, CZ, F, GR, H, RO, SK, SLO), jedoch weniger bei Ländern, die große Küstenabschnitte haben (D, DK, FIN, IRL, I, NL, PL, P, E, GB). Die Schweiz wird ausschließlich durch über die Atmosphäre importiere Schadstoffe betroffen ([7,8], persönliche Mitteilung der Autoren).

Der Vollständigkeit halber sei noch erwähnt, dass kohlegefeuerte Kraftwerke mit der Abluft auch Radioaktivität in die Umwelt freisetzen. Es handelt 
sich um Kalium sowie Uran, Thorium und Radium sowie, bei Uran und Thorium, deren radioaktive Folgeprodukte, die als Verunreinigungen in der Kohle enthalten sind. Steinkohle enthält pro kg rund 30 bis $40 \mathrm{~Bq}$ Uran, 20 bis $30 \mathrm{~Bq}$ Thorium und 40 bis $120 \mathrm{~Bq}$ Kalium-40 [21]. Bei der Verbrennung bleibt zwar der größere Teil dieser Radioaktivität in den festen Rückständen wie Asche und Schlacke zurück oder wird von den Abgasfiltern zurückgehalten. Ein kleiner Anteil gelangt in die Umwelt und trägt zur Strahlenexposition der in der Nähe lebenden Bevölkerung bei. Diese erhält dadurch zusätzliche Strahlendosen im Bereich einiger $\mu$ Sv pro Jahr (Tausendstel $\mathrm{mSv}$ ). Dies entspricht der zusätzlichen Exposition durch ein Kernkraftwerk.

\subsection{Die PSI-Studie zu den Risiken der Stromerzeugung}

Eine Arbeitsgruppe am Schweizer Paul Scherrer Institut (PSI) in Würenlingen befasst sich seit längerem mit den Risiken verschiedener Verfahren zur Stromproduktion. Die Schlussfolgerungen der 2016 veröffentlichten Studie [12] werden nachfolgend zusammengefasst. Diese Arbeit ist von großem Interesse, weil sie drei möglichen Szenarien berücksichtigt: Normalbetrieb, schwere Unfälle und, zum ersten Mal, auch Sabotage und Terrorattacken. Die Autoren betonen allerdings, dass für die Letzteren wenig Datenmaterial verfügbar sei, da Behörden und Betreiber aus verständlichen Gründen bei Informationen zu Bedrohungen sowie bei Angaben zu Maßnahmen zur Terrorabwehr sehr zurückhaltend sind. Die Studie weist darauf hin, dass bei den Auswirkungen nur Todesfälle in Betracht gezogen wurden, nicht jedoch Effekte auf die Gesundheit (Morbidität) oder auf die Umwelt. Die Arbeitsgruppe beabsichtig jedoch, dies in künftigen Studien zu berücksichtigen und diese auch auf neuere Verfahren der Stromproduktion auszuweiten. Die Resultate der Untersuchung (Tab. 5.2) werden entweder in Anzahl Todesfälle oder Anzahl verlorene Jahre an Lebenserwartung (Years of Life Lost, YOLL) angegeben, oder, erweitert durch die Anzahl der Jahre mit infolge von Krankheit verminderter Lebensqualität, als DALY-Werte (Disabilityadjusted Life Years Lost) [12].

Beim Normalbetrieb ist auch der gesamte Brennstoff- und Fabrikationszyklus eingeschlossen. Hier trägt die Wasserkraft das weitaus geringste Risiko, gemeinsam mit Wind- und Kernenergie; es folgen Photovoltaik, Geothermie und Erdgas, danach Steinkohle und Erdgas in China und schließlich mit dem höchsten Risiko die Kohlekraftwerke in China [12]. 
Tab. 5.2 PSI-Studie: Vergleich der Anzahl der Todesfälle durch verschiedene Arten der Stromerzeugung im Normalbetrieb: Angaben in YOLL/GWh relativ zu den entsprechenden Werten der Wasserkraft. (Nach [12])

\begin{tabular}{lll}
\hline Art der Stromerzeugung & Typ der Anlage & $\begin{array}{l}\text { Risiko [YOLL/GWh] } \\
\text { relativ zu Hydro }\end{array}$ \\
\hline Wasserkraft (Hydro) & Speicher-/Laufkraftwerke (CH) & 1 \\
$\begin{array}{l}\text { Windenergie } \\
\text { Kernenergie }\end{array}$ & $\begin{array}{l}\text { Onshore (CH)/Offshore (D, DK) } \\
\text { F, CH }\end{array}$ & $\approx 3$ \\
$\begin{array}{l}\text { Photovoltaik } \\
\text { Geothermie }\end{array}$ & Amorph \& kristallin (CH) & $\approx 10$ \\
Erdgas & $\mathrm{CH}$ & \\
Steinkohle & $\mathrm{CH}, \mathrm{I}$ & \\
Erdgas (GuD) & $\mathrm{D}$ & $\approx 50$ \\
Steinkohle & China & $\approx 1500$ \\
\hline
\end{tabular}

Werden nur neue und zukünftige Anlagen betrachtet, wiederum inklusive Brennstoffzyklus, dann haben die erneuerbaren Energien Wasserkraft, Wind, Sonne und Geothermie sowie die Kernenergie (EPR, [6]) bei Normalbetrieb die geringsten Auswirkungen auf Mensch und Umwelt. Von den fossilen Brennstoffen schneidet die Braunkohle am schlechtesten $a b$, nämlich mit rund 100-mal größeren Auswirkungen als die Wasserkraft; bei Erdgas sind sie noch 50-mal höher, bei der Steinkohle liegen sie zwischen den Werten von Erdgas und Braunkohle. Durch $\mathrm{CO}_{2}$-Abscheidung und Speicherung $(\mathrm{CCS}=$ Carbon Capture and Storage) würden sich die Auswirkungen der Kohleverstromung auf die Hälfte bis ein Fünftel reduzieren [12].

Für hypothetische schwere Unfälle berechnet die PSI-Studie die Anzahl der Todesfälle pro GWh sowie zusätzlich eine hypothetische Höchstanzahl von Todesfällen. Als Störfälle werden jene Ereignisse betrachtet, bei denen mindestens eines der folgenden sieben Kriterien erfüllt ist: 1) Mehr als fünf Todesfälle, 2) mehr als zehn Verletzte, 3) mehr als 200 Evakuierte, 4) Notwendigkeit von Einschränkungen beim Lebensmittelkonsum, 5) Freisetzung von mehr als 10.000 Tonnen Kohlenwasserstoff-Verbindungen, 6) Notwendigkeit zur Dekontamination von Land und Wasserflächen von mehr als $\left.25 \mathrm{~km}^{2}, 7\right)$ wirtschaftliche Verluste von über 5 Mio. US\$.

Die PSI-Studie unterscheiden zwischen Anlagen in den OECD-Ländern und solchen in Nicht-OECD-Ländern ${ }^{7}$. Insbesondere bei der Wasserkraft

\footnotetext{
${ }^{7}$ Die Organisation für wirtschaftliche Zusammenarbeit und Entwicklung OECD (Organisation for Economic Co-operation and Development) ist eine internationale Organisation mit 35 Mitgliedstaaten, die sich der Demokratie und Marktwirtschaft verpflichtet fühlen. Die meisten OECD-Mitglieder gehören zu den Ländern mit hohem Pro-Kopf-Einkommen und gelten als entwickelte Länder. http://www.oecd.org/(Zugegriffen 12.09.2019).
} 
schneiden jene in Nicht-OECD-Ländern deutlich schlechter ab, und dies gleich um mehrere Größenordnungen. Bei Kohle und Öl ist der Unterschied kleiner; beim Erdgas besteht hingegen kein Unterschied zwischen Anlagen in OECD- und Nicht-OECD-Ländern [12].

Für Anlagen in OECD-Ländern ergibt sich folgendes Bild bezüglich der Anzahl Todesfälle pro GWh nach einem schweren Unfall: Am besten schneiden westliche Kernreaktoren der Generation III+ (z. B. der EPR) $\mathrm{ab}$, gefolgt von Photovoltaik (kristallin) und solarthermischen Anlagen. An nächster Stelle stehen Geothermie, Wind Onshore (D), Wasserkraft und westliche Kernreaktoren der Generation II (PWR), gefolgt von Biogas und Wind Offshore (UK). Am höchsten ist das Risiko bei Anlagen, die mit Erdgas, Öl oder Kohle betrieben werden. Betrachtete man die hypothetischen Höchstwerte von Todesfällen nach schweren Unfällen, dann schneiden nach der PSI-Studie die erneuerbaren Energien (jedoch ohne Wasserkraft) am besten ab, gefolgt von Erdgas, Öl und Kohle; die größten Auswirkungen haben Wasserkraft (Staudammbruch) und Kernenergie [12].

Interessant ist der Vergleich zwischen Normalbetrieb und schweren Unfällen anhand der YOLL/GWh-Werte. Bei Kohle und Erdgas, aber auch bei den erneuerbaren Energien sind die Auswirkungen von schweren Unfällen um etwa zwei Größenordnungen kleiner als bei Normalbetrieb. Bei Wasserkraft gilt dies nur für die OECD-Länder; in den Nicht-OECDLändern sind die Auswirkungen eines schweren Unfalles, z. B. eines Staudammbruchs, größer als im Normalbetrieb. Bei der Kernenergie sind bei westlichen Generation-II-Reaktoren die Auswirkungen schwerer Unfälle um zwei Größenordnungen geringer als bei Normalbetrieb, bei Kernreaktoren der Generation III+ (z. B. EPR) sind sie gar um fünf Größenordnungen kleiner [12].

Für hypothetische Terroranschläge sind die Berechnungen aus den bereits genannten Gründen ungenau. Hier wurden nur Kernenergie und Wasserkraft betrachtet und bei der ersteren der Vergleich von OECD- und NichtOECD-Ländern geführt. Bei der Kernenergie wird das Risiko durch eine Terrorattacke, angegeben in YOLL/GWh, um 3 bis 4 Größenordnungen tiefer eingestuft als bei Normalbetrieb. Bei der Wasserkraft, hier am Beispiel USA und China, liegt das Risiko eines Terroranschlags auf einen großen Staudamm um eine Größenordnung höher als beim Normalbetrieb. Dieser Unterschied wird darauf zurückgeführt, dass großtechnische Anlagen (wie Kernkraftwerke) relativ gut geschützt werden können. Aus Sicht von Terroristen sind große Ansammlungen von Menschen, aber auch Staudammanlagen leichter zu attackierende Ziele als großtechnische Einrichtungen wie ein Kernkraftwerk [12]. 
Zusammenfassend lässt sich sagen, dass im Normalbetrieb die Wasserkraft das geringste Risiko aufweist und die Kernenergie verhältnismäßig gut abschneidet. Ein interessanter Unterschied besteht zwischen OECD- und Nicht-OECD-Ländern: Kohlekraftwerke und Staudammanlagen schneiden z. B. in Asien weniger gut ab, weil sowohl Sicherheitskultur als auch Wartung, Kontrollen und Nachrüstung der Anlagen noch nicht so weit entwickelt sind wie in den OECD-Ländern. Eine weitere Folgerung ist, dass die Auswirkungen insgesamt (bezogen auf die Anzahl der Todesfälle) bei Normalbetrieb größer sind als bei schweren Unfällen; bei Unfällen liegen sie in derselben Größenordnung wie bei Terror-Attacken [12].

\subsection{Risiken durch Naturkatastrophen und Epidemien}

Der Vollständigkeit halber sei noch der Bezug zwischen anthropogenen und Naturkatastrophen hergestellt, jedoch ohne Kriege und militärische Konflikte (Tab. 5.3). Die Natur, und damit auch Krankheiten und Epidemien, Missernten und Hungersnöte bildet nach wie vor das größte Gefahrenpotenzial. So haben die 20 größten Katastrophen des Jahres 2013 weltweit insgesamt 19.174 Menschenleben gefordert (SwissRe, 2014 Daten mündlich präsentiert an der Davoser Tagung [16]). Durch das Erdbeben von 2010 in Haiti starben 222.570 Menschen (International Research Institute of Disaster Science, Tōhoku University, Japan, May 2014). Der Tsunami vom zweiten Weihnachtstag 2004 in Südostasien forderte mehr als 230.000 Menschenleben. Auf der International Desaster and Risk Conference 2014 in Davos [16] wurde die Vermutung geäußert, dass die Zahl der Toten durch den Tsunami nach dem Tōhoku-Erdbeben von Fukushima im März 2011 möglicherweise höher ist als die von den Behörden gemeldete offizielle Zahl von 18.784 Opfern. ${ }^{8}$

Nach den Risikostatistiken von Versicherungskonzernen ist eine Zunahme von großen Schadensereignissen festzustellen. Dies hängt zum einen mit dem Anstieg der Weltbevölkerung zusammen und der besseren Erfassung solcher Ereignisse. Ein Teil der Zunahme dürfte jedoch auf den Klimawandel zurückzuführen sein.

\footnotetext{
${ }^{8}$ Die diesem Abschnitt zitierten Zahlenwerte wurden an der International Desaster and Risk Conference vom 14.-18. März 2014 in Davos in Vorträgen mündlich präsentiert [16].
} 
Tab. 5.3 Epidemien, Krankheiten und deren Folgen [26]

\begin{tabular}{llll}
\hline Jahr & Was & Opfer & Wo \\
\hline 1918-1920 & Spanische Grippe & bis 50 Mio. Tote & Weltweit \\
1957-1958 & Asiatische Grippe & 1-2 Mio. Tote & Weltweit \\
1968-1970 & Hongkong-Grippe & 1 Mio. Tote & Weltweit \\
Seit 1980 & HIVIAids & 36 Mio. Tote & Weltweit \\
2009-2010 & Schweinegrippe & 18.449 Tote & Weltweit \\
Seit 2010 & Cholera & 8.942 Tote & Haiti \\
Seit Dezember 2013 & Chikungunay-Fieber & 875.000 Erkrankte & Zentral- und \\
& & & Südamerika \\
Feb. 2014-Mrz. 2016 & Ebola-Fieber & 11.000 Tote & Afrika \\
Seit Dezember 2019 & COVID-19 & 233.000 (WHO 1.5.20) & Weltweit \\
\hline
\end{tabular}

\section{Katastrophenstatistiken (Zusammengestellt aus [28])}

«... Größere Versicherungskonzerne führen in der Regel geographisch organisierte Risikostatistiken, die ihnen als Berechnungsgrundlage für Versicherungsprämien dienen. Die EM-DAT International Disasters Database der Weltgesundheitsorganisation dokumentiert seit 1888 die weltweiten Katastrophen. Demnach ereigneten sich zwischen 1900, 2000 und 2003 insgesamt 9195 größere Katastrophen mit jeweils mindestens 10 Toten. Davon hatten Wetterkatastrophen mit $57 \%$ den höchsten Anteil, keine $20 \%$ waren geologischen Ursprungs (Vulkanausbrüche, Erdbeben), wie auch die in die geologische Kategorie gezählten Tsunamis; der Rest waren biologische Katastrophen (Seuchen und Plagen)...»

«... Die Weltbank hat 2005 in ihrem Report „Natural Disaster Hotspots: A Global Risk Analysis“ Karten publiziert, die die Verteilung der Risiken auf Weltkarten zeigen. Etliche davon sind zu sehen auf den Seiten der Columbia University. Nach dem 2016 erschienenen Bericht der Weltbank waren in der Dekade 2005 bis 2014 durchschnittlich 17 Mio. Menschen pro Jahr von Naturkatastrophen betroffen; in der Dekade 1976-1985 waren es noch 60 Mio. Menschen gewesen. Zugleich verzehnfachten sich die dabei entstandenen Kosten von 14 auf mehr als 140 Mio. US\$ pro Jahr. Durch die menschengemachte Globale Erwärmung und die starke Zunahme der Weltbevölkerung bzw. der Bevölkerungsdichte in vielen Regionen der Welt werden in Zukunft deutlich mehr Menschen von Naturkatastrophen betroffen sein als früher. Wenn keine Schutzmaßnahmen (d. h. Klimaschutz und Anpassung an die globale Erwärmung) getroffen würden, könnten bis 2050 ca. 1,3 Mrd. Menschen durch Naturkatastrophen bedroht werden und sich die bis dahin entstehenden Kosten auf 158 Billionen US\$ belaufen. Dies ist etwa das Doppelte des derzeitigen Weltsozialproduktes. Die in den besonders betroffenen Küstenstädten anfallenden Schadenskosten könnten von $6 \mathrm{Mrd}$. \$ im Jahr 2010 auf eine Billion Dollar im Jahr 2070 ansteigen...»

«... In diese Kategorie der EU (größter Teil Bevölkerung betroffen, schwere und dauerhafte Auswirkungen auf Lebensbedingungen und wirtschaftliche Stabilität) fallen etwa ein Dutzend Ereignisse der letzten 100 Jahre in Europa, von denen die Hitzewelle 2003 mit 70.000 Todesopfern und das Elbehochwasser 2002 mit Schäden in Höhe von etwa $18 \mathrm{Mrd}$ € als verheerendste zu verzeichnen sind. Weltweit gehen solche Ereignisse in diesem Zeitraum in die Hunderte; als teuerste bezifferte Katastrophen der Geschichte gelten das Erdbeben von Kōbe 1995 und der Hurrikan Katrina 2005 mit bis zu 100 Mrd. US\$ volkswirtschaftlichem Schaden. Was die opferreichste Naturkatastrophe der Geschichte ist, lässt sich kaum sagen; zu nennen wären die Dürren in Indien 1965-1967 mit an die 1,5 Mio. Toten und die Überschwemmungen in Indien 1955 mit 45 Mio. Obdachlosen - über frühe Ereignisse liegen seltener Angaben über Opferzahlen vor und kaum monetäre Schätzungen. ...» 


\subsection{Risiken der globalen Erwärmung}

Die Auswirkungen des Klimawandels werden immer deutlicher spürbar und können zu einer globalen Bedrohung für die Menschheit werden [17, 19, 20]. Zwar wirken sich die natürlicherweise in der Atmosphäre vorhandenen Treibhausgase positiv aus für das Leben auf der Erde, denn ohne diese läge die durchschnittliche Temperatur der Atmosphäre um $33^{\circ} \mathrm{C}$ tiefer. Das Klimasystem, das sich vor der industriellen Ära noch in einem Gleichgewicht befand, ist seither durch die Emissionen von anthropogenen Treibhausgasen erheblich gestört worden. Das Treibhausgas $\mathrm{CO}_{2}$ liefert den größten Beitrag zum Klimawandel. Der weltweite jährliche Ausstoß aus menschlichen Tätigkeiten betrug im Jahr 1900 noch 2 Gt, stieg bis 1950 auf 5 Gt und lag 2012 bereits bei 35,5 Gt. Addiert man die andern Treibhausgase, kommt man auf 46,6 Gt $\mathrm{CO}_{2}$-Äquivalent. ${ }^{9,10}$

Es wird prognostiziert, dass Häufigkeit und Intensität von extremen Wettersituationen wie Hitzewellen, Stürme etc. in den kommenden Jahren infolge des Klimawandels zunehmen werden. Der Hitzesommer 2003 forderte in Europa zwischen 35.000 und 70.000 Todesopfer und versursachte volkswirtschaftliche Schäden im Bereich von 13 Mrd. US\$ [13]. Die danach vorbereiteten Notfallmaßnahmen mussten bereits 2015 aktiviert werden. In mehreren Ländern wird bereits eine klimabedingte Übersterblichkeit festgestellt, und man schätzt, dass die durch Stürme und Unwetter verursachten Schäden Kosten im Milliardenbereich (Euro) nach sich ziehen [14].

Der IPCC-Bericht: Human Health: Impacts, Adaptation, and CoBenefits gibt folgende mögliche gesundheitliche Konsequenzen des Klimawandels [18]:

- Höheres Verletzungs-, Krankheits- und Todesrisiko durch stärkere Hitzewellen und Brände (Wahrscheinlichkeit: hoch),

- Erhöhtes Risiko für Unterernährung infolge verminderter Nahrungsmittelproduktion in armen Regionen (Wahrscheinlichkeit: hoch),

- Folgen für die Gesundheit von Arbeitsunfähigkeit und verminderter Arbeitsproduktivität in gefährdeten Bevölkerungsgruppen (Wahrscheinlichkeit. hoch),

\footnotetext{
${ }^{9}$ Globale Treibhausgas-Emissionen [ $\mathrm{CO}_{2}$-Äquiv.] in Prozent: Energie und Heizen: 25; Land- und Forstwirtschaft sowie Landnutzung: 24, Bau: 6, Transport 14, Industrie 21 übrige 10, [11, 18].

${ }^{10}$ Der neueste Bericht des IPCC „Emission Gap Report 2018“ spricht für das Jahr 2017 von rund 50 $\mathrm{Gt} \mathrm{CO}_{2}$-Äquivalent (ohne Beitrag durch Landnutzungsänderung) bzw. rund $53.5 \mathrm{Gt} \mathrm{CO}_{2}$-Äquivalent inklusive Landnutzungsänderung (Land Use Change) [32].
} 
- Erhöhte Risiken für durch Lebensmittel und Wasser übertragene Krankheiten (Wahrscheinlichkeit: sehr hoch) und durch Vektoren übertragene Krankheiten (Wahrscheinlichkeit: hoch),

- Geringe Reduktion der durch Kälte bedingten Mortalität und Morbidität in einigen Gebieten aufgrund weniger kalter Extreme (Wahrscheinlichkeit: gering) und geografischer Verschiebungen,

- in der Nahrungsmittelproduktion verringerte Kapazität von krankheitsübertragenden Vektoren aufgrund der Überschreitung der thermischen Schwellenwerte (Wahrscheinlichkeit: mittel).

- Die positiven Auswirkungen (des Klimawandels) werden weltweit durch das Ausmaß und die Schwere der negativen Auswirkungen des Klimawandels (Wahrscheinlichkeit: hoch) überkompensiert.

\section{Auch die EU macht sich Gedanken über die Folgen des Klimawandels}

Eine im Auftrag der EU durchgeführte Studie einer Forschergruppe des Joint Research Centre in Ispra (I) unter der Leitung von Giovanni Forzieri hat den Einfluss des Klimawandels auf das Wetter in Europa untersucht und dessen Folgen für die menschliche Gesundheit.

"We found that weather-related disasters could affect about two-thirds of the European population annually by the year 2100 (351 Mio. people exposed per year [uncertainty range 126 to 523 Mio.] during the period 2071-2100), compared with $5 \%$ during the reference period (1981-2010; 25 Mio. people exposed per year). About 50 times the number of fatalities occurring annually during the reference period (3000 deaths) could occur by the year 2100 (152.000 deaths [CI: 80.500-239.800]). Future effects show a prominent latitudinal gradient, increasing towards southern Europe, where the premature mortality rate due to weather extremes (about 700 annual fatalities per million inhabitants [CI: 482-957] during the period 2071-2100 vs. 11 during the reference period) could become the greatest environmental risk factor. The projected changes are dominated by global warming (accounting for more than $90 \%$ of the rise in risk to human beings), mainly through a rise in the frequency of heatwaves (about 2700 heat-related fatalities per year during the reference period vs 151.500 [CI: 80.100-239.000] during the period 2071-2100)....

Global warming could result in rapidly rising costs of weather-related hazards to human beings in Europe unless adequate adaptation measures are taken. Our results could aid in prioritisation of regional investments to address the unequal burden of effects on human beings of weather-related hazards and differences in adaptation capacities.» [10]

\section{Zunehmende Waldbrandgefahr in Südeuropa}

Die Gebiete mit erhöhtem Waldbrandrisiko vergrößern sich vor allem in Südeuropa (Spanien, Portugal, Südfrankreich und Korsika, Italien und Griechenland). Mit hoher Wahrscheinlichkeit ist das eine Folge des Klimawandels; auch die Anzahl betroffener Wohngebiete und die Anzahl der Opfer steigt jedes Jahr. 2017 hat sich das Risiko gegenüber früheren Jahren verdreifacht und die Periode mit erhöhter Waldbrandgefahr in diesen Regionen hat sich von Juli bis August auf die Monate Juni bis Oktober ausgedehnt. Es ist zu befürchten, dass künftig weitere Gebiete in Europa betroffen sein werden [27]. 


\section{Mögliche Auswirkungen des Klimawandels}

"In den Polargebieten: Bedrohung der Artenvielfalt und Schrumpfung der Tundren und Auftauen der Permafrostböden

In den kalt-gemäßigten Klimazonen: Vermehrte Waldbrände, Insektenbefall und Ausbreitung von Infektionskrankheiten

In den kühl-gemäßigten Klimazonen: Vermehrte Waldbrände, Insektenbefall und Krankheiten

In den kontinentalen Mittelbreiten (Weizenanbaugebiete): Dürren im Sommer, Destabilisierung der Ökosysteme mit drastischen Folgen für die menschliche Nutzung. Demgegenüber wird Weinanbau in Großbritannien möglich und in Südeuropa können Dattelpalmen und Agaven genutzt werden.

In den Subtropen: Die dichtbevölkerten Regionen der halbtrockenen Subtropen (u. a. das Mittelmeergebiet, der Südwesten der USA, der Norden Mexikos, der Süden Australiens und Afrikas und Teile Südamerikas) werden vermutlich noch trockener werden.

In den Tropen: Zum einen dürften die halbtrockenen Tropen (z. B. die Savannen des Sahel) von zunehmenden Niederschlägen profitieren, sodass der Ackerbau mehr Erträge bringt. Die feuchten Zonen der Tropen, die bereits weitgehend entwaldet sind, werden hingegen durch zunehmende Trockenheit und Waldbrände weiter ihre Artenvielfalt einbüßen. Intakter Regenwald hingegen wirkt ausgleichend auf den Wasserhaushalt und kommt mit steigenden Temperaturen relativ gut klar.» (sinngemäß zitiert aus [9])

Weitere mögliche Folgen sind Versauerung und Erwärmung der Meere; Erhöhung des Meeresspiegels, Veränderung der Meeresströme (z. B. des Golfstroms); Ergrünen der Sahara; Rückgang und Verschwinden der Gletscher; Abschmelzen der Polkappen und Eisschilde; Veränderungen der Niederschlagsmengen und Abflussregime der Flüsse; Dürren und Überschwemmungen; Zunahme von Häufigkeit und Stärke von tropischen Wirbelstürmen; Waldbrände; Rückgang der Biodiversität und der Artenvielfalt; Auftauen der Permafrostböden; Methanfreisetzungen aus den Methanhydraten in den Meeresböden; schließlich auch politische und wirtschaftliche Folgen; Flüchtlingsströme; gesundheitliche Folgen und Epidemien; volkswirtschaftliche Schäden etc. ${ }^{11}$

\footnotetext{
${ }^{11}$ Weiterführende Informationen: Akademien der Wissenschaft Schweiz 2012 und 2017 sowie die auf ins Deutsche übersetzten Berichte des Intergovernmental Panel on Climate Change [19].
} 


\section{Die Sonne, unser Energielieferant und der Treibhauseffekt der Erde [35]}

Die auf der Erde direkt messbare Größe ist die Energie der Sonneneinstrahlung, angegeben in Watt pro $\mathrm{m}^{2}$ senkrecht zur Strahlrichtung. Sie wird Solarkonstante genannt und beträgt $S=1367 \mathrm{~W} / \mathrm{m}^{2}$. Mit dem Abstandsgesetz und der Annahme, dass die Sonne nach allen Seiten gleichmäßig strahlt, lässt sich die Leuchtstärke der Sonne berechnen $\left(L_{\odot}=3,846 \cdot 10^{26} \mathrm{~W}\right)$ sowie die Intensität der Strahlung an der Sonnenoberfläche $\left(I_{\odot}=6,49 \cdot 10^{7} \mathrm{~W} / \mathrm{m}^{2}\right)$. Mithilfe des Stefan-Bolzmann-Gesetzes $\left(I=\sigma \cdot \mathrm{T}^{4}\right)$ kann die Temperatur der Sonnenoberfläche, der Photosphäre, bestimmt werden: $T_{P h \odot}=5780 \mathrm{~K} \approx 5500{ }^{\circ} \mathrm{C}$. Hier ist $\sigma$ die Stefan-Boltzmann-Konstante und $\mathrm{T}$ die absolute Temperatur in Kelvin.

Das Sonneninnere ist noch wesentlich heißer, nämlich rund 15,6 $10^{6} \mathrm{~K}$. Dort erzeugt die Sonne ihre Energie durch Fusion von je vier Protonen zu einem Helium-Kern. Man nennt dies die pp-Reaktion. Bei der Umwandlung von Wasserstoff zu Helium dient das schwere Wasserstoff Deuterium $\left({ }^{2} \mathrm{H}\right)$ als Zwischenstufe, indem zwei Protonen durch die Reaktion $\mathrm{p}+\mathrm{e}^{-} \rightarrow$ $\mathrm{n}+\nu_{\mathrm{e}}$ und $\mathrm{p}+\mathrm{n} \rightarrow{ }^{2} \mathrm{H}$ in Neutronen umgewandelt werden. Die Energiebilanz der pp-Reaktion beträgt $26,35 \mathrm{MeV}$. Dabei entstehen auch die Sonnenneutrinos $\left(v_{\mathrm{e}}\right)$, von denen jede Sekunde rund $65 \mathrm{Mrd}$. pro $\mathrm{cm}^{2}$ auf der Erdoberfläche auftreffen.

Die Sonne als Fusionsreaktor verliert durch diesen Energieerzeugungsprozess jede Sekunde 4,3 Mio. Tonnen ihrer Masse sowie eine weitere Million Tonnen durch den Sonnenwind. Diese Massenabnahme macht allerdings, bezogen auf die ganze «Lebensdauer»der Sonne, also das rund $11 \mathrm{Mrd}$. Jahren andauernde Wasserstoffbrennen, nur gerade mal ein Promille der gewaltigen Sonnenmassen von $M_{\odot}=1,988 \cdot 10^{30} \mathrm{~kg}$ aus.

Die von der Erde absorbierte Sonnenenergie beträgt

$$
P_{a}=(1-r) \pi \cdot R_{E^{2}} \cdot S=1,36 \cdot 10^{17} \mathrm{~W}
$$

Hier ist $S$ ist die Solarkonstante, $R_{E}$ der Erdradius und $r$ der Anteil der von Erdoberfläche und Atmosphäre reflektierten Strahlung (Albedo) von rund 30 \%. Die von der Sonne erwärmte Erde strahlt wiederum nach dem Stefan-Boltzmann-Gesetz Energie ab, aber jetzt im IR-Bereich:

$$
P_{E}=4 \pi \cdot R_{E}^{2} \cdot e \cdot \sigma \cdot T_{E}^{4}
$$

Hier ist $e$ der «Wirkungsgrad» dieser Rückstrahlung mit $e \approx 0,6$. Er berücksichtigt jenen Anteil der Erdstrahlung, der nicht von den Treibhausgasen in der Atmosphäre zurückgehalten wird.

Im Gleichgewichtszustand gilt $P_{a}=P_{E}$ sowie

$$
P_{a, e f f}=\frac{1}{4}(1-r) \cdot S=e \cdot T_{E}^{4}=240 \mathrm{~W} / \mathrm{m}^{2},
$$

denn die Erdoberfläche emittiert in diesem Fall genauso viel Energie wie sie von der Sonne erhält, allerdings nicht im gleichen Wellenlängenbereich. Hieraus ergibt sich für die Erdoberfläche eine durchschnittliche Temperatur von $T_{E}=289 \mathrm{~K}=+15^{\circ} \mathrm{C}$. Würde dieselbe Rechnung für $e=1$ gemacht, dann wäre die Atmosphäre für die IR-Strahlung der Erde völlig durchlässig und es ergäbe sich eine deutlich tiefere Durchschnittstemperatur der Erdoberfläche mit $T_{E}=256 \mathrm{~K}=-18^{\circ} \mathrm{C}$.

Die natürlichen Treibhausgase, zu denen neben $\mathrm{CO}_{2}$ auch Wasserdampf, Methan und Ozon gehören, bewirken somit, dass es auf der Erde rund $33^{\circ} \mathrm{C}$ wärmer ist. Mit diesem Wissen lässt sich erahnen, was passiert, wenn der Mensch dieses natürliche Gleichgewicht durch die Emission zusätzlicher Treibhausgase nachhaltig verändert. 


\section{Literatur}

1. BFS (2015) Schweizerischer Krebsbericht 2015: Stand und Entwicklungen. Bundesamt für Statistik, Neuenburg

2. BFS (2017) Todesursachenstatistik 2015. https://www.bfs.admin.ch/bfs/de/ home.gnpdetail.2017-0081.html. Zugegriffen: 20. Juli 2019

3. BFS (2018). https://www.bfs.admin.ch/bfs/de/home/statistiken/bevoelkerung. html. Zugegriffen: 14. Nov. 2017

4. Cardis E et al (2006) Estimates of the cancer burden in Europe from radioactive fallout from the Chernobyl accident. Int J Cancer 119(6):1224-1235

5. Conca J (2012) How deadly is your Kilowatt? We Rank Killer Energy Sources. Forbes, 10. Juni. 2013

6. EPR European Pressurized Water Reactor. https://de.wikipedia.org/wiki/Areva_ EPR. Zugegriffen: 1. Nov. 2017

7. Europe's Dark Cloud (2016a) Europe's dark cloud - how coal-burning countries are making their neighbours sick. Autoren. In: Jones D, Huscher J, Myllyvitra L, Gierens R, Flisowska J, Gutmann K, Urbaniak D and Azau S. (Hrsg) WWF | Climate action network Europe | Health and environment alliance | sandbag (Supported by the European Union)

8. Europe's Dark Cloud (2016b) Lifting Europe's dark cloud - how cutting coal saves lives autoren. In: Schaible C, Lazarus A, Jones D, Filsowska J, Huscher $\mathrm{J}$ and Urbaniak D. (Hrsg) European environmental Bureau |Clean action network Europe | HEAL | Sandbag and WWF (Supported by the European Union)

9. Folgen der globalen Erwärmung. https://de.wikipedia.org/wiki/Folgen_der_ globalen_Erw\%C3\%A4rmung. Zugegriffen: 7. Aug. 2017

10. Forzieri $G$ et al (2017) Increasing risk over time of weather-related hazards ot he European population: a data-driven prognostic study. Lancet Planet Health 1:E200-e208

11. Global Carbon Atlas (2019) CO2 emissions. http://www.globalcarbonatlas.org/ en/CO2-emissions. Zugegriffen: 20. Febr. 2019

12. Hirschberg $S$ et al (2016) Health effects of technologies for power generation: contributions from normal operation, severe accidents and terrorist threat health effects. Reliab Eng Sys Saf 145:373-387

13. Hitzewelle in Europa (2003). https://de.wikipedia.org/wiki/Hitzewelle_in_ Europa_2003. Zugegriffen: 4. Aug. 2017

14. Hitzewelle in Europa (2015). https://de.wikipedia.org/wiki/Hitzewellen_in_ Europa_2015. Zugegriffen: 4. Aug. 2017

15. ICRP (2007) The 2007 recommendations of the International Commission on Radiological Protection. Ann ICRP 2007 37(2-4):1-332 
16. IDRC: 5th International Disaster and Risk Conference IDRC Davos 2014: integrative risk management - the role of science, technology \& practice, 24-28 August 2014, Davos/CH, Organised by GRF Davos in close cooperation with the United Nations Office for Disaster Risk Reduction (UNISDR). (oral presentations).

17. IPCC The Intergovernmental Panel on Climate Change. https://www.ipcc.ch/. Zugegriffen: 8. Aug. 2017

18. IPCC (2014a) Contribution of working group III to the fifth assessment report of the intergovernmental panel on climate change

19. IPCC (2014b) Intergovernmental Panel on Climate Change (2013/14): Klimaänderung 2013/2014: Zusammenfassung für politische Entscheidungsträger, Herausgeber: Deutsche IPCC-Koordinationsstelle/DLR Projektträger, mit dem Österreichische Umweltbundesamt und ProClim (von ScNat) für die Schweiz. ProClim, Bern

20. IPCC (2017) Berichte zum Klimawandel und dessen Auswirkungen. http:// www.ipcc.ch/. Zugegriffen: 9. Aug. 2017

21. Jansen D (2008) Radioaktivität aus Kohlekraftwerken. Bund (Freunde der Erde) Bund-Hintergrund, Berlin

22. Katastrophe von Bhopal. http://de.wikipedia.org/wiki/Katastrophe_von_Bhopal. Zugegriffen: 3. Aug. 2017

23. Liste von Stauanlagenunfällen. http://de.wikipedia.org/wiki/Liste_von_Stauanlagenunf\%C3\%A4llen. Zugegriffen: 3. Aug. 2017

24. Liste von Flugunfällen. https://de.wikipedia.org/wiki/Liste_von_Flugunf\%C3\%A4llen_ab_2011\#2015. Zugegriffen: 11. Sept. 2019

25. Liste von Unglücken im Bergbau. https://de.wikipedia.org/wiki/Liste_von_ Ungl\%c3\%BCcken_im_Bergbau\#cite_note-80. Zugegriffen: 3. Aug. 2017

26. Listen von Epidemien und Pandemien. https://de.wikipedia.org/wiki/Liste_ von_Epidemien_und_Pandemien. Zugegriffen: 4. Aug. 2017

27. Modugno S, Balzter H, Cole B, Borrelli P (2016) Mapping regional patterns of large forest fires in wildland-urban interface areas in Europe. J Environ Manag 172(1):112-126

28. Naturkatastrophe. https://de.wikipedia.org/wiki/Naturkatastrophe. Zugegriffen: 4. Aug. 2017

29. OECD (2018) The full costs of electricity provision. NEA Nr. 2798, OECD/ NEA, Boulogne-Billancourt

30. Renn O (2014) Das Risikoparadox - Warum wir uns vor dem Falschen fürchten. Fischer Taschenbuch, Frankfurt a. M.

31. Spaeth A (2018) Noch nie war das Fliegen so sicher wie 2017. NZZ, 3. Januar, Zürich

32. UN Environnent: Emission Gap Report (2018). https://wedocs.unep.org/bitstream/handle/20.500.11822/26895/EGR2018_FullReport_EN.pdf?sequen$\mathrm{ce}=1$ \&isAllowed $=\mathrm{y}$. Zugegriffen: 11. Sept. 2019 
33. UVEK (2017) Eidgenössisches Departement für Umwelt, Verkehr, Energie und Kommunikation UVEK: Kenngrössen zur Entwicklung der Treibhausgasemissionen in der Schweiz, 1990-2015. Bundesamt für Umwelt, Ittigen/CH

34. Verkehrstod. https://de.wikipedia.org/wiki/Verkehrstod. Zugegriffen: 20. Febr. 2019

35. Völkle H (2013) Die Sonne - unser Stern. Bull Soc Frib Sc Nat 102:85-106

36. de Sanctis E, Monti S und Ripani M (2016) Energy from nuclear fission. Springer International Publishing Switzerland, Cham 